\title{
CONTRIBUIÇÃO PARA O ESTUDO DA COMPOSIÇÃO E DIGESTIBILIDADE DO CAPIM JARAGUÁ (Hiparrhenia Rufa (Nees) Stapf.)
}
W. R. JARDIM
C. L. MORAES
A. M. PEIXOTO
Prof. Cated.
Assist.
Assist.

INDICE

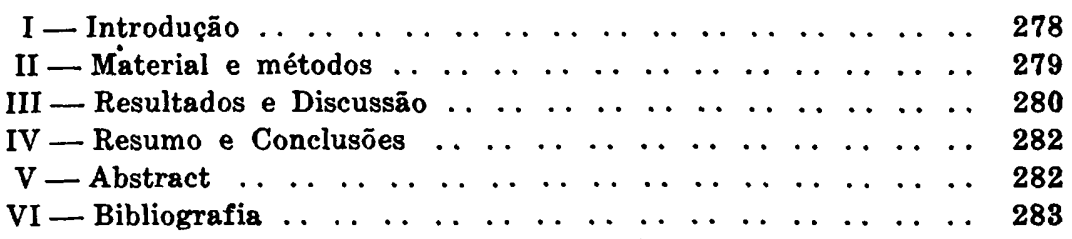

* Trabalho apresentado e aprovado na II Reunião Interamericana da Produção Animal - Baurú, Est. São Paulo - 1952. 


\section{I - INTRODUÇÃO}

Os rumos atuais da bromatologia, em face da crescente dificuldade na obtenção de resíduos industriais de alto nível protêico, voltam-se mais uma vez para o aproveitamento das forragens grosseiras, procurando nos pastos, fenos e alimentos ensilados, a chave da solução para o problema da alimentação dos dos animais. Inúmeros dados estatísticos de paises cuja pecuária já atingiu alto grau de desenvolvimento, mostram que, ultimamente, tem havido um apreciável aumento das áreas destinadas aos pastos, prados e capineiras, em detrimento do consumo de concentrados. Estes fatos assumem uma importância muito grande para o nosso país, onde, em virtude da extensão de seu território e variedade das condiçóes ecológiças, prosperam as mais diversas espécies forrageiras, ainda tão mal conhetidas e aproveitadas.

๑ estudo do valor nutritivo de nossas plantas forrageiras está quase todo por realizar. Embora possamos reunir numerosas observaçōes sôbre a :composição química (1) dessas forrageiras, faltam-nos aindà os dados sôbré a sua digestibilidade pelos animais, o que constitui sem dúvida, uma grande falha, dada a importância que isso representa dentro do problema do correto arraçoamento. Mesmo as gramíneas consideradas clásricas nos pastos do Brasil Central, os capins gordura, jaraguá e coloniấo, estão a exigir ainda dos técnicos, pesquizas mais profundàs, a fim de se aquilatar o seu real valor.

KOK e colaboradores (2,3 e 4) iniciaram em 1942, no Departamento da Produção Animal do Estado de São Paulo alguns ensaios de digestibilidade com forrageiras nacionais e exóticas, os quais vếm sendo continuados por LEME DA ROCHA e outros $(5,6)$. Porém, a messe de resultados colhidos é ainda pequena, em face da extensão do campo a pesquizar. Há irealmente, necessidade de um extenso trabalho, cujos dados possam revelar o verdadeiro valor nutritivo das plantas nativas e estrangeiras cultivadas em nosso ambiente para a alimentação dos animais.

Neste trabalho, os autores apresentam uma contribuição ao estudo do valor nutritivo do capim Jaraguá (Hyparrhenia rufa, (Nees) Stapf.), considerado nas condições do Estado de São Paulo, uma das gramíneas mais interessantes, tanto para o gado de leite, como para o de engorda. Originário do planalto central, em Goiaz e Mato Grosso, onde cresce expontaneamente, 6 "tapim jaraguá disseminou-se por outros estados do Brasil. Em São Paulo é; depois do gordura, a gramínea mais usada na 
formação de pastagens, dominando principalmente, na zona de terra roxa do estado, onde encontra na fertilidade do solo, condições muito favoráveis para o seu desenvolvimento. Não encontramos, todavia, em nossa pesquiza bibliográfica, tanto no país como no estrangeiro (7), dados suficientemente seguros, sốbre a digestibilidade dessa importante gramínea. Assim, procurando realizar um estudo mais completo da forrageira em questão, os autores ensaiaram a sua digestibilidade em vários estágios de desenvolvimento, e nas formas de feno e silagem.

\section{II - MATERIAL E MÉTODOS}

O capim jaraguá, no estado verde, foi estudado em três estágios diferentes : novo, em plena floração e depois da floração (produzindo sementes). O material, fornecido pela Fazenda Modêlo, da E. S. A. "Luiz de Queiroz", proveio de terra roxa de baixada, não adubada, tendo o capim, aproximadamente, $45 \mathrm{~cm}, 135 \mathrm{~cm}$ e $140 \mathrm{~cm}$, de altura, nos três estágios citados, respectivamente.

Para a produção de feno, o Jaraguá foi cortado antes da floração, com $60 \mathrm{~cm}$ de altura, fenado ao sol e conservado em medas. A silagem, obtida em silo tipo "trincheira", foi feita com capim jaraguá também cortado antes da floração, com aproximadamente $80 \mathrm{~cm}$ de altura, e retirada após três meses e meio, para análise e distribuição aos animais. A operação de ensilagem e os cuidados posteriores obedeceram à boa técnica aconselhada.

Os ensaios de digestibilidade foram realizados com carneiros, devidamente arreiados e mantidos em baias individuais, de acôrdo com os métodos seguidos por MEAD e GUILBERT (8), bem como por KOK e colaboradores (2). As experiências foram levadas a efeito com lotes de 4 ou 5 animais, para as várias formas de forrageiras, como será esclarecido adiante. Em cada ensaio foram considerados dois períodos : um preparatório, de 8 dias, e outro, principal, de 7. O critério de 7 dias para o período principal foi adotado não só para facilitar o trabalho, mas também porque os seus resultados são perfeitamente satisfatórios, como demonstraram os ensaios de STAPLES e DINNUSSON (9) e L'HARPE e colaboradores (10).

$O$ capim verde, o feno e a silagem foram picados e pesados diàriamente, para as rações, sendo retiradas e dessecadas todos os dias, amostras para a análise. Em cada ensaio foram realizadas três análises, tanto dos limentos como das fezes. Estas, colhidas também diàriamente, foram sêcas a $100^{\circ} \mathrm{C}$, pesadas e moi- 
das, retirando-se $1 / 10$ da quantidade produzida por indivíduo. Os coeficientes de digestibilidade foram obtidos com base nos resultados das análises das forragens e das fezes, na matéria sêca a $100^{\circ} \mathrm{C}$. guintes :

Os métodos seguidos nas análises químicas foram os se-

1) Umidade - Secagem em estufa a $100^{\circ} \mathrm{C}$, até pêso constante;

2) Cinza - Incineração em mufla a $550^{\circ} \mathrm{C}$, até a obtenção de cinza bem clara;

3) Matéria graxa - Extração com éter, usando-se extrator de Soxhlet, segundo A.O.A.C., pag. 408 (11);

4) Proteina - Dosagem do nitrogênio orgânico e amoniacal segundo Kjeldahl, modificado por Gunning e Arnold (A.O.A.C.), e multiplicado por 6,25;

5) Fibra - Segundo A.O.A.C., pg. 409;

6) Extrativos não nitrogenados - Determinação por diferença;

7) Cálcio - Dosagem nas cinzas, segundo A.O.A.C., pg. 119; 22.

8) Fósforo - Dosagem nas cinzas, segundo A.O.A.C., pg.

\section{III - RESULTADOS E DISCUSSÃO}

1) Capim Jaraguá Novo

Animais : 4 carneiros Romney-Marsh

Ração : 1.800 gramas por dia e por cabeça

Período preparatório: 8 dias (11-11-51 a 18-11-51)

Período principal : 7 dias (19-11-51 a 25-11-51)

(Quadros I, II, III e IV)

2) Capim Jaraguá em plena floração

Animais : 5 carneiros Romney-Marsh

Ração: 1.500 gramas por dia e por cabeça

Periodo preparatório: 8 dias (13-5-52 a 20-5-52)

Período principal : 7 dias (21-5-52 a 27-5-52)

(Quadros V, VI, VII e VIII) 
3) Capim jaraguá depois da floração (produzindo sementes)

Animais : 5 carneiros Romney-Marsh

Ração: 1.200 gramas por dia e por cabeça

Periodo preparatório: 8 dias (9-6-52 a 16-6-52)

Periodo principal: 7 dias (17-6-51 a 23-6-52)

(Quadros IX, X, XI e XII)

4) Feno de capim jaraguá

Animais : 4 carneiros Romney-Marsh

Ração: 600 gramas por dia e por cabeça

Período preparatório : 9 dias (17-5-51 a 25-5-51)

Período principal: 6 dias (27-5-51 a 1-6-51)

(Quadros XIII, XIV, XV e XVI)

5) Silagem de capim jaraguá

Animais : 4 carneiros Romney-Marsh

Ração: 1.000 gramas por dia e por cabeça

Periodo preparatório : 8 dias (9-9-51 a 16-9-51)

Período principal: 7 dias (17-9-51 a 23-9-51)

(Quadros XVII, XVIII, XIX e XX)

A maturidade econômica constitui um dos pontos mais importantes no estudo das forrageiras. Mediante a determinação da digestibilidade dos vários nutrientes, em diversos estágios de desenvolvimento da forrageira, é possível estabelecer a melhor época para se efetuar o corte, ou introduzir os animais na pastagem. De modo geral, o teor de proteina e minerais é mais elevado e o de fibra mais baixo, quando as plantas são novas. $O$ teor de proteina e minerais diminui até a maturação, e depois, mais ainda, durante a produção de sementes. E' o que demonstra o quadro XXI, onde se acham resumidos os resultados dos nossos ensaios com o capim em estado verde. Os nutrientes digestiveis do Jaraguá, nos 3 estágios de desenvolvimento considerados, são postos em confronto, e pode se verificar perfeitamente o valor nutritivo muito mais alto para a gramínea quando nova. A queda do teor de proteina é bastante apreciável, o que determina um alargamento muito grande da relação nutritiva, que de 1:8,8 passa a 1:94. Os teores de cálcio e fósforo também se reduzem bastante, em mais de $50 \%$ a medida que a planta crèsce, embora a relação $\mathrm{CaO}: \mathrm{P} 2 \mathrm{O}$, mantenha-se mais ou menos estável.

O teor de fibra digestível mais alto no Jaraguá em plena floração, do que no capim após a floração (produzindo semen: 
tes), é provàvelmente devido à transformação da celulose em lignina (componente indigestível), como se depreende da composição em fibra bruta (Quadros II, VI, e X) da forrageira.

O quadro XXII mostra resultados de ensaios com fenos, realizados no Dep. da Produção Animal de S. Paulo e na 5a. Cadeira da E. S. A. "Luiz de Queiroz". As forrageiras utilizadas pelo D. P. A. foram cultivadas em sua maioria em terreno adubado, o que não aconteceu com as amostras da "Luiz de Queiroz".

O teor de proteina no feno de Jaraguá, embora baixo comparativamente, pode ser considerado satisfatório, nas condições em que o capim vegetou, isto é, no pasto sem adubação.

Embora o Jaraguá não seja muito recomendado para a ensilagem, por ser pouco suculento, o produto obtido é superior à silagem de milho, obtida nas mesmas condições, em nutrientes digestíveis totais, e foi bem aceito pelos animais. $O$ quadro XXIII, mostra o resultado das comparações realizadas.

\section{IV - RESUMO e CONCLUSOES}

Os autores apresentam neste trabalho os resultados dos ensaios realizados no Laboratório de Bromatologia da 5a. Cadeira da Escola Superior de Agricultura "Luiz de Queiroz", através dos quais estudaram a composição química e o valor nutritivo do capim Jaraguá, em diversos estágios de desenvolvimento, e sob as formas de feno e silagem.

As experiências de digestibilidade foram feitas com carneiros, segundo a técnica recomendada por MEAD E GUILBERT. (8) e as análises realizadas conforme os métodos químicos usualmente empregados, como é esclarecido no texto. XXV.

Os resultados acham-se resumidos nos auadros XXIV e

\section{V - ABSTRACT}

The authors repport in this paper, the results of trials by means of which were determined the composition and nutritive value of Capim Jaraguá, Hyparrhenia rufa (Nees) Stapf. cultivated in Piracicaba, in State of São Paulo, Brazil. 
The experiments were made with hay, silage and also the grass at three different stages : before, during and after blooming.

The digestion trials were carried out with sheep, following the technique of MEAD and GUILBERT. The chemical analysis of the forages were made by methods usually employd.

The results are in the table XXIV e XXV of this paper.

\section{VI - BIBLIOGRAFIA}

1. BOLLigeR, R. - Analyses de Forragens. Publicação do Instituto Agrônomico do Estado. Campinas. 1930.

2. KOK, E. A., ANDRADE, B. M. e MACHADO, L. B. - O Capim de Rhodes - Bol. Ind. Animal. Vol. 5, n. 1-2: 39-53. São Paulo. 1943.

3. KOK, E. A., MACHADO, L. B. e MEIRELLES, L. V. - Valor nutritivo de plantas forrageiras - Bol. Ind. Animal. Vol. 6, n. 4: 67-83. São Paulo. 1943.

4. KOK, E. A., MACHADO, L. B. e ROCHA, G. L. - Valor nutritivo de plantas forrageiras. Bol. Ind. Animal. Vol. 8, n. 3: 18-44. São Paulo. 1946.

5. ROCHA, G. L., MACHADO, L.B. BOTELHO, F. B. e CORREA, H. S. - Ensaios de digestibilidade (aparente) do Capim Catingueiro Roxo - Melinis minutiflora. Pal de Beauv. Bol. Ind. Animal. Vol. 12, n. único : 107-117. São Paulo. 1951.

6. ROCHA, G. L., BECKER, M. BOTELHO, F. B. e CORREA, N. S. - Ensaios de digestibilidade (aparente) de plantas forrageiras - Bol. Ind. Animal. Vol. 12, n. único: 119-129. São Paulo. 1951.

7. SCHNEIDER, B. H. - Feeds of the world - Agricultural Experiment Station, West Virginia University, Morganton. 1947.

8. MEAD, S. M. e GUILBERT, H. R. - The digestibility of certain fruit by products as determined for ruminants. Bull 409. University of califonia. Berkeley. 1926. 
9. STAPLES, G. E. e DINUSSON, W. R. - A comparison of relative accuracy between seven-day and ten-day collection periods in digestion trials-Jour. Animal Science. Vol. 10, n. 1:244-250. New York. 1951.

10. L'HARPE, J. DE, PINEYRUA, J. e SUSACQ, J. B. - Investigacion sobre digestibilidad - Anais do II Congresso Rio Grandense de Agronomia. 1.o Vol. 203-216. Rio Grande do Sul. 1940.

11. A. O. A. C. - Official and tentative methods of Analysis of the Association of Official Agricultural Chemists (A.O.A.C.) - 6a. Edition. Washington. D. C. 1945.

12. JARDIM, W. R., MORAES, C. L. e PEIXOTO, A. M. - Contribuição para o estudo da composição e valor nutritivo de plantas forrageiras. I parte - Anais da Escola Superior de Agricultura "Luiz de Queiroz". 32-38. Piracicaba. 1952.

13. HODGSON, H. E. e REED, O. E. - Manual de Lacticínios para a América Tropical Publicação T. C. - 290. Washington. D. C. 1951. 
Quadro I : Total de alimentos consumidos e fezes coletadas

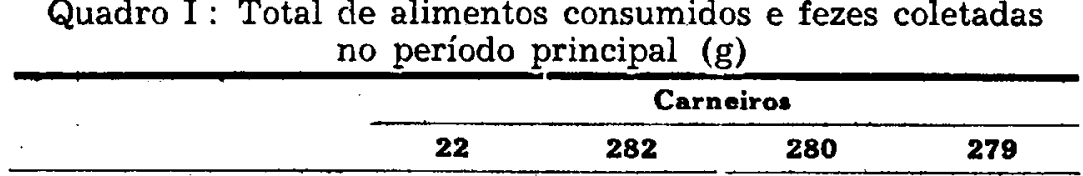

\begin{tabular}{lrrrr}
\hline $\begin{array}{l}\text { Ração total consumi- } \\
\text { da }\end{array}$ & 10.693 & 10.668 & 7.832 & 10.704 \\
$\begin{array}{l}\text { Ração total consumi- } \\
\text { da (sêca a 100 }\end{array}$ & 3.180 & 3.173 & 2.329 & 3.183 \\
$\begin{array}{l}\text { Fezes coletadas } \\
\text { Fezes coletadas (sê- }\end{array}$ & 2.725 & 2.938 & 2.801 & 3.139 \\
cas a 100 $100^{\circ} \mathrm{C}$ ) & 1.345 & 1.345 & 1.120 & 1.301 \\
\hline
\end{tabular}

Quadro II : Análises químicas do capim Jaraguá novo e das fezes (Médias de todas as análíses)

\begin{tabular}{|c|c|c|c|c|c|c|c|c|c|c|c|c|c|c|c|c|}
\hline & \multicolumn{7}{|c|}{$\mathrm{Na}$ matéria sêca a $100^{\circ} \mathrm{C}(\%)$} & \multicolumn{9}{|c|}{ Na matéria úmida (\%) } \\
\hline & Prot. & M. G. & Fibra & E. N. N. & Cinza & $\mathrm{CaO}$ & P2O5 & Umid. & M. S. & Prot. & M. G. & Fibra & E. N. N & Cinze & $\mathrm{CaO}$ & $\mathbf{P 2 O 5}$ \\
\hline $\begin{array}{l}\text { Capim Jaraguá novo } \\
\text { Fezes do carneiro } 22 \\
\text { Fezes do carneiro } 282 \\
\text { Fezes.do carneiro } 280 \\
\text { Fezes.do carneiro } 279\end{array}$ & $\begin{array}{l}9,18 \\
8,15 \\
8,34 \\
7,99 \\
9,06\end{array}$ & $\begin{array}{l}2,62 \\
2,56 \\
2,73 \\
2.57 \\
2,74 \\
\end{array}$ & $\begin{array}{l}28,87 \\
23,99 \\
25,54 \\
25,49 \\
27,31\end{array}$ & $\begin{array}{l}44,38 \\
39,67 \\
36,67 \\
39,08 \\
35,75 \\
\end{array}$ & $\begin{array}{l}14,95 \\
25,63 \\
26,72 \\
24,87 \\
25,14 \\
\end{array}$ & $\begin{array}{l}0,56 \\
=\end{array}$ & $\begin{array}{l}0,45 \\
= \\
=\end{array}$ & $\begin{array}{l}70,26 \\
50,64 \\
54,22 \\
60,01 \\
58,57\end{array}$ & $\begin{array}{l}29,74 \\
49.36 \\
45,78 \\
39,99 \\
41,43\end{array}$ & $\begin{array}{l}2,73 \\
4,02 \\
3,81 \\
3,19 \\
3,75\end{array}$ & $\begin{array}{l}0,77 \\
1,26 \\
1,24 \\
1,01 \\
1,13\end{array}$ & $\begin{array}{r}8,58 \\
11,84 \\
11,69 \\
10,19 \\
11,31\end{array}$ & $\begin{array}{l}13,22 \\
19,59 \\
16,81 \\
15,66 \\
14,83\end{array}$ & $\begin{array}{r}4,44 \\
12,65 \\
12,23 \\
9,94 \\
10,41 \\
\end{array}$ & $\begin{array}{l}0,16 \\
= \\
=\end{array}$ & $\begin{array}{l}0,12 \\
=\end{array}$ \\
\hline
\end{tabular}

Quadro III : Coeficientes de digestibilidade do capim Jaraguá novo $(\%)$

\begin{tabular}{lccccc}
\multicolumn{5}{c}{ Jaraguá novo $(\%)$} \\
\cline { 2 - 6 } & Prot. Bruta & Mat. Graxa & Fibra & E. N. N & Mat. Sêca \\
\hline Carneiro 22 & 62,44 & 58,67 & 64,85 & 62,19 & 57,70 \\
Carneiro 282 & 61,48 & 55,83 & 63,96 & 64,97 & 57,60 \\
Carneiro 280 & 58,14 & 52,83 & 57,54 & 57,75 & 51,91 \\
Carneiro 279 & 59,68 & 57,27 & 61,35 & 67,08 & 59,14 \\
\hline Média & 60,43 & 56,15 & 61,92 & 62,99 & 56,58 \\
\hline
\end{tabular}

Quadro IV: Valor nutritivo do capim Jaraguá novo (\%)

\begin{tabular}{lccc}
\hline & Nutrientes brutos & $\begin{array}{c}\text { Coeficientes do } \\
\text { digestibilidado }\end{array}$ & $\begin{array}{c}\text { Nutrientes } \\
\text { dizostiveis }\end{array}$ \\
\hline Umidade & 70,26 & & \\
Matéria sêca & 29,74 & 56,58 & 16,82 \\
Proteina & 2,73 & 60,43 & 1,64 \\
Matéria graxa & 0,77 & 56,15 & 0,43 \\
Fibra & 8,58 & 61,92 & 5,31 \\
E. N. Nitrogen. & 13,22 & 62,99 & 8,32 \\
\hline \multicolumn{2}{c}{ Nutrientes digestíveis totais } & & 16,23 \\
Relação nutritiva & & $1: 8,8$ \\
Relação CaO: P2O5 & & $1: 0,75$ \\
\hline
\end{tabular}


Quadro V: Total de alimentos consumidos e fezes coletadas no período principal $(\mathrm{g})$

\begin{tabular}{|c|c|c|c|c|c|}
\hline & \multicolumn{5}{|c|}{ Carneiros } \\
\hline & 36 & 33 & 30 & 34 & 28 \\
\hline $\begin{array}{l}\text { Ração total consu- } \\
\text { mida }\end{array}$ & 8.195 & 8.764 & 7.664 & 7.832 & 6.270 \\
\hline $\begin{array}{l}\text { Ração total consu- } \\
\text { mida (sêca a } 100^{\circ} \mathrm{C} \text { ) } \\
\text { Fezes coletadas }\end{array}$ & $\begin{array}{l}2.808 \\
3.164\end{array}$ & $\begin{array}{l}3.003 \\
3.309\end{array}$ & $\begin{array}{l}2.626 \\
2.698\end{array}$ & $\begin{array}{l}2.683 \\
2.890\end{array}$ & $\begin{array}{l}2.148 \\
2.181\end{array}$ \\
\hline $\begin{array}{l}\text { Fezes coletadas (sê- } \\
\text { cas a } 100^{\circ} \mathrm{C} \text { ) }\end{array}$ & 1.449 & 1.552 & 1.479 & 1.404 & 1.198 \\
\hline
\end{tabular}

Quadro VI : Análises químicas do capim jaraguá em plena floração e das fezes (Média de todas as análises)

\begin{tabular}{|c|c|c|c|c|c|c|c|c|c|c|c|c|c|c|c|c|}
\hline & \multicolumn{7}{|c|}{ Na matéria séca a $100^{\circ} \mathrm{C}(\%)$} & \multicolumn{9}{|c|}{ Na matéria úmida (\%) } \\
\hline & Prot. & M. G. & Fibra & E. N. N. & Cinza & $\mathrm{CaO}$ & P205 & Umid. & M. S. & Prot. & M. G. & Fibra & E. N. N & Cinza & $\mathrm{CaO}$ & P2O5 \\
\hline $\begin{array}{ll}\text { Capim Jaraguá em plen: } \\
\text { floração } \\
\text { Fezes do carneiro } & 36 \\
\text { Fezes do carneiro } & 33 \\
\text { Fezes do carneiro } & 30 \\
\text { Fezes do carneiro } & 34 \\
\text { Fezes do carneiro } & 28\end{array}$ & $\begin{array}{l}3,48 \\
5,16 \\
4,42 \\
4,41 \\
4,25 \\
4,15\end{array}$ & $\begin{array}{l}1,91 \\
1,94 \\
1,91 \\
1,98 \\
1,98 \\
2,15\end{array}$ & $\begin{array}{l}31,43 \\
26,45 \\
26,84 \\
26,67 \\
26,78 \\
26,71\end{array}$ & $\begin{array}{l}49,57 \\
40,62 \\
42,67 \\
42,87 \\
40,94 \\
42,93 \\
\end{array}$ & $\begin{array}{l}13,61 \\
25,83 \\
25,82 \\
24,07 \\
26,05 \\
24,06\end{array}$ & $\begin{array}{r}0,27 \\
= \\
=\end{array}$ & $\begin{array}{r}0,17 \\
= \\
=\end{array}$ & $\begin{array}{l}65,74 \\
54,22 \\
53,10 \\
44,19 \\
51,43 \\
45,06 \\
\end{array}$ & $\begin{array}{l}34,26 \\
45,78 \\
46,90 \\
54,81 \\
48,57 \\
54,94 \\
\end{array}$ & $\begin{array}{l}1,19 \\
2,36 \\
2,07 \\
2,41 \\
2,06 \\
2,28 \\
\end{array}$ & $\begin{array}{l}0,65 \\
0,88 \\
0,89 \\
1,08 \\
0,96 \\
1,18 \\
\end{array}$ & $\begin{array}{l}10,76 \\
12,10 \\
12,58 \\
14,61 \\
13,00 \\
14,61 \\
\end{array}$ & $\begin{array}{l}17,00 \\
18,62 \\
19,26 \\
23,52 \\
19,90 \\
23,66 \\
\end{array}$ & $\begin{array}{r}4,66 \\
11,82 \\
12,10 \\
13,19 \\
12,65 \\
13,21 \\
\end{array}$ & $\begin{array}{r}0,09 \\
= \\
= \\
\end{array}$ & $\begin{array}{r}0,05 \\
= \\
=\end{array}$ \\
\hline
\end{tabular}

\begin{tabular}{lccccc}
\multicolumn{6}{c}{ Quadro VII : Coeficientes de digestibilidade do capim } \\
jaraguá em plena floraçäo $(\%)$ \\
\hline & Prot. Bruta & Mat. Graxa & Fibra & E. N. N. & Mat. Sêca \\
\hline Carneiro 36 & 23,50 & 45,74 & 56,57 & 57,71 & 48,40 \\
Carneiro 33 & 19,47 & 48,34 & 55,85 & 55,49 & 48,30 \\
Carneiro 30 & 28,63 & 41,73 & 52,21 & 51,29 & 43,68 \\
Carneiro 34 & 21,07 & 45,76 & 55,40 & 47,56 & 47,68 \\
Carneiro 28 & 33,48 & 37,20 & 52,59 & 51,69 & 44,21 \\
\hline Média & 25,23 & 43,75 & 54,52 & 52,74 & 46,45 \\
\hline
\end{tabular}

Quadro VIII : Valor nutritivo do capim jaraguá

\begin{tabular}{lccc} 
& Nutrientes brutos & $\begin{array}{c}\text { Coeficientes de } \\
\text { digestibilidade }\end{array}$ & $\begin{array}{c}\text { Nutrientes } \\
\text { digestíveis }\end{array}$ \\
\hline Umidade & & & \\
Matéria sêca & 65,74 & - & \\
Proteina & 34,26 & 46,45 & 15,91 \\
Matéria graxa & 1,19 & 25,23 & 0,30 \\
Fikra & 0,65 & 43,75 & 0,28 \\
E. N Nitrogen. & 10,76 & 54,52 & 5,86 \\
\hline \multicolumn{2}{c}{ Nutrientes digestiveis totais } & & 8,96 \\
Relaçãa nutritiva & & 15,75 \\
Relação CaO : P2O5 & & $1: 51$ \\
\hline
\end{tabular}


Quadro IX: Total de alimentos consumidos e fezes coletadas no periodo principal $(g)$

\begin{tabular}{lrrrrr}
\hline & \multicolumn{5}{c}{ Carneiros } \\
\cline { 2 - 6 } & $\mathbf{3 6}$ & $\mathbf{2 8}$ & $\mathbf{3 4}$ & $\mathbf{2 8 0}$ & $\mathbf{2 7 9}$ \\
\hline Ração total consu- & & & & & \\
$\quad$ mida & 7.960 & 5.333 & 7.667 & 6.490 & 7.995 \\
$\begin{array}{l}\text { Ração total consu- } \\
\text { mida (sêca a 100 }\end{array}$ & 2.827 & 1.894 & 2.723 & 2.305 & 2.839 \\
$\begin{array}{l}\text { Fezes coletadas } \\
\text { Fezes coletadas (sê- }\end{array}$ & 4.002 & 2.411 & 3.974 & 4.492 & 4.439 \\
$\quad$ cas a 100 & & & & \\
\hline
\end{tabular}

Quadro X: Análises químicas do capim jaraguá, depois da floração, (produzindo sementes) e das fezes coletadas (Médias de todas as análises)

\begin{tabular}{|c|c|c|c|c|c|c|c|c|c|c|c|c|c|c|c|c|}
\hline & \multicolumn{7}{|c|}{ Na matéria séca a $100^{\circ} \mathrm{C}(\%)$} & \multicolumn{9}{|c|}{ Na matéria úmida (\%) } \\
\hline & Prot. & M. G. & Fibra & E. N. N. & Cinzz & $\mathrm{CaO}$ & P2O5 & Umid. & M. $\mathbf{s}$. & Prot. & M. G. & Fibra & E. N N. & Cinza & $\mathrm{CaO}$ & P2O5 \\
\hline $\begin{array}{l}\text { Capim Jaraguá produzido } \\
\text { sementes } \\
\text { Fezes do carneiro } 36 \\
\text { Fezes do carneiro } 28 \\
\text { Fezes do carneiro } 34 \\
\text { Fezes do carneiro } 280 \\
\text { Fezes do carneiro } 279\end{array}$ & $\begin{array}{l}2,80 \\
4,21 \\
4,28 \\
3,93 \\
3,91 \\
4,11\end{array}$ & $\begin{array}{l}1,56 \\
1,61 \\
1,58 \\
1,48 \\
1,56 \\
1,64\end{array}$ & $\begin{array}{l}33,67 \\
30,54 \\
31,86 \\
30,15 \\
31,11 \\
31,91\end{array}$ & $\begin{array}{l}50,47 \\
43,54 \\
41,39 \\
46,19 \\
45,26 \\
42,42 \\
\end{array}$ & $\begin{array}{l}11,50 \\
20,10 \\
20,89 \\
18,25 \\
18,16 \\
19,87\end{array}$ & $\begin{array}{l}0,25 \\
= \\
=\end{array}$ & $\begin{array}{l}0,16 \\
= \\
=\end{array}$ & $\begin{array}{l}64,49 \\
60,23 \\
60,02 \\
56,96 \\
68,92 \\
64,45\end{array}$ & $\begin{array}{l}35,51 \\
39,77 \\
39,98 \\
43,04 \\
31,08 \\
35,55\end{array}$ & $\begin{array}{l}0,99 \\
1,67 \\
1,71 \\
1,69 \\
1,21 \\
1,46\end{array}$ & $\begin{array}{l}0,55 \\
0,64 \\
0,63 \\
0,76 \\
0,48 \\
0,58\end{array}$ & $\begin{array}{r}11,95 \\
12,14 \\
12,73 \\
12,97 \\
9,66 \\
11,34\end{array}$ & $\begin{array}{l}17,94 \\
17,33 \\
16,56 \\
19,77 \\
14,09 \\
15,11\end{array}$ & $\begin{array}{l}4,08 \\
7,99 \\
8,35 \\
7,85 \\
5,64 \\
7,06\end{array}$ & $\begin{array}{r}0,09 \\
= \\
=\end{array}$ & $\begin{array}{r}0,05 \\
= \\
=\end{array}$ \\
\hline
\end{tabular}

Quadro XI : Coeficientes de digestibilidade do capim jaraguá produzindo sementes $(\%)$

\begin{tabular}{lccccc}
\hline \multicolumn{7}{c}{ jaragua produzindo sementes $(\%)$} \\
\hline & Prot. Bruta & Mat. Graxa & Fibra & E. N. N. & Mat. Sêca \\
\hline Carneiro 36 & 15,32 & 41,89 & 49,96 & 51,41 & 43,68 \\
Carneiro 28 & 22,16 & 48,44 & 51,81 & 58,23 & 49,07 \\
Canneiro 34 & 11,00 & 40,40 & 43,73 & 42,59 & 37,16 \\
Carneiro 280 & 15,42 & 39,41 & 44,02 & 45,67 & 39,42 \\
Carneiro 279 & 18,41 & 41,55 & 47,23 & 53,28 & 44,41 \\
\hline Média & 16,46 & 42,33 & 47,35 & 50,23 & 42,74 \\
\hline
\end{tabular}

Quadro XII : Valor nutritivo do capim jaraguá produzindo sementes $(\%)$

Coeficientes do Nutrientes

\begin{tabular}{lccc}
\hline & Nutrientes brutos & $\begin{array}{c}\text { Coeficientes de } \\
\text { digestibilidade }\end{array}$ & $\begin{array}{c}\text { Nutrientes } \\
\text { digeativeis }\end{array}$ \\
\hline Umidade & 64,49 & & \\
Matéria sêca & 35,51 & & \\
Proteina & 0,99 & 16,74 & 15,17 \\
Matéria graxa & 0,55 & 42,46 & 0,16 \\
Fibra & 11,95 & 47,35 & 0,23 \\
E. N. Nitrogen. & 17,92 & 50,23 & 5,65 \\
\hline Nutrientes digestíveis totais & & 9,00 \\
Relação nutritiva & & 15,32 \\
Relação $\mathrm{CaO}:$ P2O5 & & $1: 94$ \\
\hline
\end{tabular}


Quadro XIII : Total de alimentos consumidos e fezes coletadas no período principal (g)

\begin{tabular}{|c|c|c|c|c|}
\hline & \multicolumn{4}{|c|}{ Carneiros } \\
\hline & 22 & 282 & 280 & 279 \\
\hline \multirow{4}{*}{$\begin{array}{l}\text { Ração total consu- } \\
\text { mida } \\
\text { Ração total consu- } \\
\text { mida (sêca a } 100^{\circ} \mathrm{C} \text { ) } \\
\text { Fezes coletadas } \\
\text { Fezes coletadas (sê- } \\
\text { cas a } 100^{\circ} \mathrm{C} \text { ) }\end{array}$} & 2.238 & 2.421 & 2.107 & 2.757 \\
\hline & 1.932 & 2019 & & \\
\hline & 1.845 & 2.362 & 2.662 & 2.691 \\
\hline & 957 & 1.048 & 941 & 1.059 \\
\hline
\end{tabular}

Quadro XIV : Análises químicas do feno de jaraguá e das fezes coletadas (Médias de todas as análises)

\begin{tabular}{|c|c|c|c|c|c|c|c|c|c|c|c|c|c|c|c|c|}
\hline & \multicolumn{7}{|c|}{$\mathrm{Na}$ matéria séca a $100^{\circ} \mathrm{C}(\%)$} & \multicolumn{9}{|c|}{$\mathrm{Na}$ matéria úmida $(\%)$} \\
\hline & Prot. & M. G. & Fibra & E. N. N. & Cinza & $\mathrm{CaO}$ & P205 & Umid. & M. s. & Prot. & M. G. & Fibra & E. N. N. & Cinza & $\mathrm{CaO}$ & P2O5 \\
\hline $\begin{array}{l}\text { Feno de Jaraguá } \\
\text { Fezes do carneiro } 22 \\
\text { Fezes do carneiro } 282 \\
\text { Fezes do carneiro } 280 \\
\text { Fezes do carneiro } 279 \\
\end{array}$ & $\begin{array}{l}6,52 \\
5,85 \\
5,61 \\
5,92 \\
6,01\end{array}$ & $\begin{array}{l}2,32 \\
2,20 \\
2,20 \\
2,31 \\
2,37 \\
\end{array}$ & $\begin{array}{l}35,02 \\
32,81 \\
33,90 \\
33,91 \\
30,96\end{array}$ & $\begin{array}{l}38,24 \\
29,11 \\
27,26 \\
26,79 \\
30,89 \\
\end{array}$ & $\begin{array}{l}17,90 \\
30,03 \\
31,03 \\
31,07 \\
29,77\end{array}$ & $\begin{array}{l}0,61 \\
=\end{array}$ & $\begin{array}{r}0,46 \\
=\end{array}$ & $\begin{array}{l}13,66 \\
48,09 \\
53,04 \\
64,65 \\
60,61 \\
\end{array}$ & $\begin{array}{l}86,34 \\
51,91 \\
46,96 \\
35,35 \\
39,39\end{array}$ & $\begin{array}{l}5,63 \\
3,03 \\
2,64 \\
2,09 \\
2,36 \\
\end{array}$ & $\begin{array}{l}2,01 \\
1,14 \\
1,03 \\
0,81 \\
0,93\end{array}$ & $\begin{array}{l}30,21 \\
16,63 \\
15,91 \\
11,98 \\
12,18 \\
\end{array}$ & $\begin{array}{r}33,03 \\
15,51 \\
12,81 \\
9,49 \\
12,20 \\
\end{array}$ & $\begin{array}{l}15,46 \\
15,58 \\
14,57 \\
10,98 \\
11,72 \\
\end{array}$ & $\begin{array}{r}0,53 \\
= \\
\end{array}$ & $\begin{array}{l}0,40 \\
= \\
=\end{array}$ \\
\hline
\end{tabular}

Quadro XV: Coeficientes de digestibilidade do feno de jarag uá (\%)

Prot. Bruta Mat. Graxa Fibra E. N. N. Mat. Sêca

\begin{tabular}{lllllll}
\hline Carneiro 22 & 55,53 & $\mathbf{5 3 , 0 0}$ & $\mathbf{5 3 , 5 7}$ & $\mathbf{6 2 , 2 7}$ & 50,44 \\
Carneiro 282 & 55,25 & $\mathbf{5 0 , 7 8}$ & 49,74 & 62,99 & 48,08 \\
Carneiro 280 & 53,03 & 48,50 & 49,91 & 63,76 & 48,27 \\
Carneiro 279 & $\mathbf{5 8 , 9 5}$ & 54,50 & $\mathbf{6 0 , 6 3}$ & 64,03 & $\mathbf{5 5 , 4 8}$ \\
\hline Média & 55,69 & 51,69 & 53,46 & $\mathbf{6 3 , 2 6}$ & 50,57 \\
\hline
\end{tabular}

Quadro XVI : Valor nutritivo de feno de jaraguá (\%) Nutrientes brutos Coeficientes de Nutrientes
digestiveis

\begin{tabular}{|c|c|c|c|}
\hline & Nutrientes brutos & $\begin{array}{l}\text { digstibilidade } \\
\text { digstions }\end{array}$ & digestiveis \\
\hline $\begin{array}{l}\text { Umidade } \\
\text { Matéria sêca } \\
\text { Proteina } \\
\text { Matéria graxa } \\
\text { Fibra } \\
\text { E. N. Nitrogen. }\end{array}$ & $\begin{array}{r}13,66 \\
86,34 \\
5,63 \\
2,01 \\
30,21 \\
33,03\end{array}$ & $\begin{array}{l}\overline{50,57} \\
55,69 \\
51,69 \\
53,46 \\
63,26\end{array}$ & $\begin{array}{r}\overline{43,66} \\
3,13 \\
1,03 \\
16,15 \\
20,89 \\
\end{array}$ \\
\hline \multicolumn{3}{|c|}{$\begin{array}{l}\text { Nutrientes digestíveis totais } \\
\text { Relaçãa nutritiva } \\
\text { Relação } \mathrm{CaO}: \mathrm{P} 2 \mathrm{O} 5\end{array}$} & $\begin{array}{c}42,48 \\
1: 12,6 \\
1: 0,75\end{array}$ \\
\hline
\end{tabular}


Quadro XVII : Total de alimentos consumidos e fezes coletadas no período principal (g)

\begin{tabular}{lrrrr}
\hline & \multicolumn{4}{c}{ Carneiros } \\
\cline { 2 - 3 } & $\mathbf{2 2}$ & $\mathbf{2 8 2}$ & $\mathbf{2 8 0}$ & \multicolumn{2}{c}{$\mathbf{2 7 9}$} \\
\hline Ração total consu- & & & & \\
$\quad$ mida & 4.845 & 4.375 & 3.881 & 5.181 \\
$\begin{array}{l}\text { Ração total consu- } \\
\quad \text { mida (sêca a 100 }\end{array}$ & 1.561 & 1.409 & 1.243 & 1.668 \\
$\begin{array}{l}\text { Fezes coletadas } \\
\text { Fezes coletadas (sê- }\end{array}$ & 2.324 & 1.954 & 2.195 & 2.712 \\
$\quad$ cas a 100 & & & \\
\end{tabular}

Quadro XVIII : Análises químicas da silagem de capim jaraguá e das fezes coletadas (Médias de todas as análises)

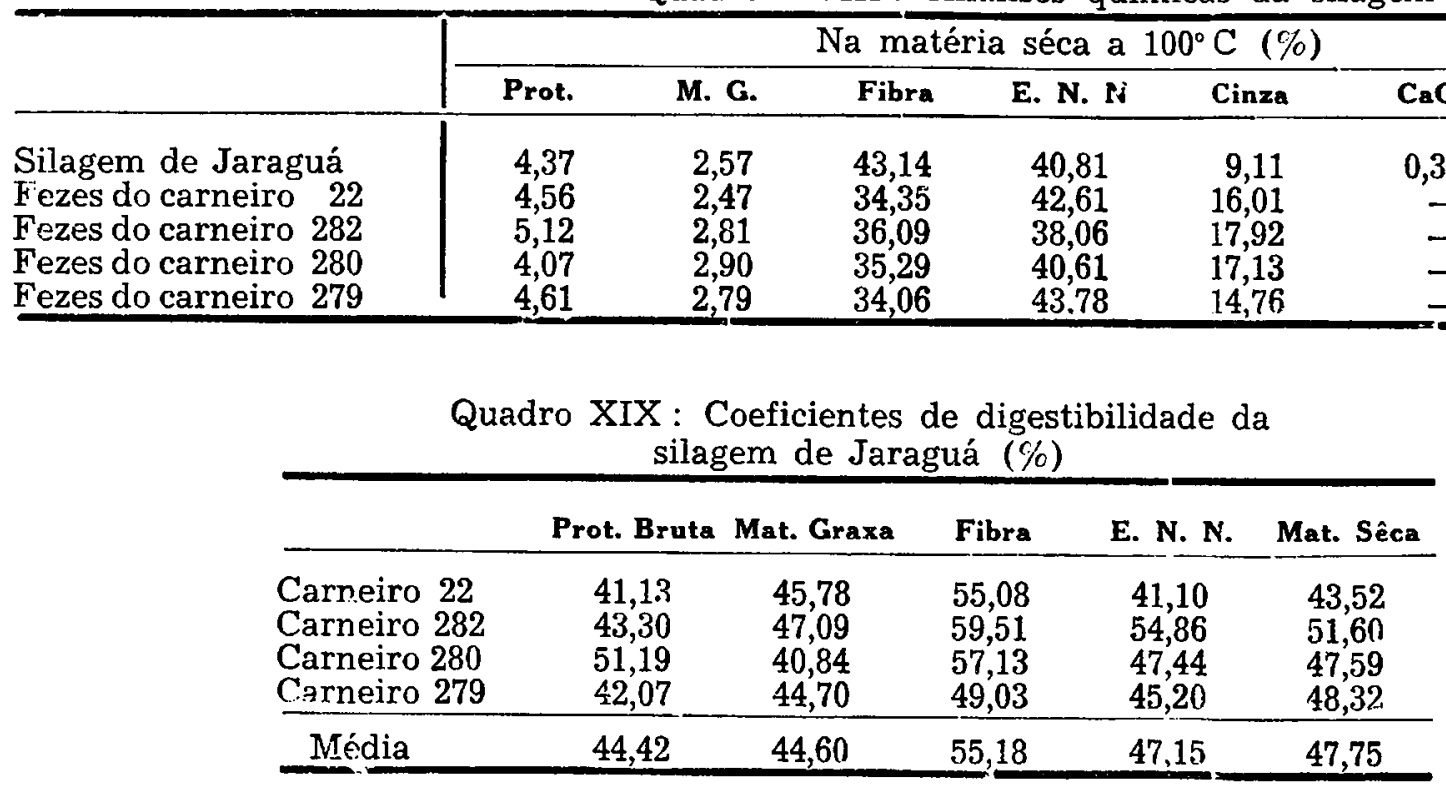

\begin{tabular}{|c|c|c|c|c|c|c|c|c|}
\hline \multicolumn{9}{|c|}{$\mathrm{Na}$ matéria úmida (\%) } \\
\hline Umid. & M. $\mathrm{s}$. & Prot. & M. G. & Fibra & E. N. N. & Cinza & $\mathrm{CaO}$ & P2O5 \\
\hline 67,80 & 32,20 & 1,40 & 0,82 & 13,89 & 13,16 & 2,93 & 0,12 & 0,06 \\
\hline 62,11 & 37,89 & 1,72 & 0,93 & 13,01 & 16,17 & 6,06 & - & - \\
\hline 65,11 & 34,89 & 1,78 & 0,98 & 12,59 & 13,29 & 6,25 & 一 & 一 \\
\hline 70,32 & 29,68 & 1,20 & 0,86 & 10,77 & 11.77 & 5,08 & 一 & 一 \\
\hline 68,58 & 31.42 & 1,44 & 0,87 & 10,70 & 13,78 & 4,63 & $=$ & - \\
\hline
\end{tabular}

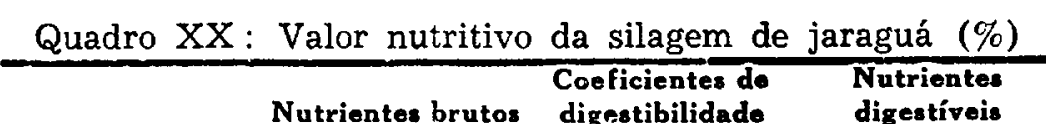

\begin{tabular}{lrrr} 
Umidade & 67,80 & & \\
Matéria sêca & 32,30 & 47,75 & 15,37 \\
Proteina & 1,40 & 44,42 & 0,62 \\
Matéria graxa & 0,86 & 44,60 & 0,36 \\
Fibra & 13,89 & 55,18 & 7,66 \\
E. N. Nitrogen. & 13,16 & 47,15 & 6,20 \\
\hline \multicolumn{2}{c}{ Nutrientes digestíveis totais } & & 15,29 \\
Relação nutritiva & & $1: 23,6$ \\
Relação CaO : P2O5 & & $1: 0,50$ \\
\hline
\end{tabular}


Quadro XXI : Nutrientes digestiveis do capim jaraguá (estado verde)

\begin{tabular}{|c|c|c|c|c|c|c|c|c|c|c|}
\hline \multirow{2}{*}{$\begin{array}{l}\text { Capim } \\
\text { Jaraguá }\end{array}$} & \multicolumn{5}{|c|}{$\begin{array}{c}\text { Nutrientes digestiveis } \\
\text { (na materia sêca) }\end{array}$} & \multicolumn{3}{|c|}{$\mathrm{Na}$ matória sêca } & \multirow{2}{*}{ N. D. T. } & \multirow{2}{*}{ R. N. } \\
\hline & M. s. & Prot. & M. G. & Fibra & E. N. N. & Cinza & $\mathrm{CaO}$ & P2O5 & & \\
\hline $\begin{array}{l}\text { Novo } \\
\text { P. F. } \\
\text { D. F. }\end{array}$ & $\begin{array}{l}16,82 \\
15,91 \\
15,17\end{array}$ & $\begin{array}{l}1,64 \\
0,30 \\
0,16\end{array}$ & $\begin{array}{c}0,43 \\
0,28 \\
0,23\end{array}$ & $\begin{array}{l}5,31 \\
5,86 \\
5,65 \\
\end{array}$ & $\begin{array}{l}8,32 \\
8,96 \\
9,00 \\
\end{array}$ & $\begin{array}{l}14,95 \\
13,61 \\
11,50\end{array}$ & $\begin{array}{r}0,45 \\
.0,17 \\
0,16\end{array}$ & $\begin{array}{l}0,56 \\
0,27 \\
0,25\end{array}$ & $\begin{array}{l}16,23 \\
15,75 \\
15,32\end{array}$ & $\begin{array}{l}1: 8,8 \\
1: 51 \\
1: 94\end{array}$ \\
\hline
\end{tabular}

Quadro XXII : Quadro comparativo dos resultados obtidos em ensaios com fenos

\begin{tabular}{|c|c|c|c|c|c|c|c|}
\hline \multirow{2}{*}{ Fenos } & \multirow{2}{*}{$\begin{array}{c}\text { Nutriente: } \\
\text { Prot. }\end{array}$} & \multirow{2}{*}{$\begin{array}{c}\text { digestiveis } \\
\text { M. G. } \\
\end{array}$} & \multicolumn{2}{|c|}{ na matéria sêca (\%) } & \multirow{2}{*}{ N. D. T. } & \multirow{2}{*}{ R. N. } & \multirow{2}{*}{$\begin{array}{c}\text { Estágio de } \\
\text { desenvolvi- } \\
\text { mento }\end{array}$} \\
\hline & & & Frbra & E. N. N. & & & \\
\hline $\begin{array}{l}\text { Rhodes } \\
\text { Colonião } \\
\text { Elefante bras. } \\
\text { Kikuiu } \\
\text { Grama Paulista } \\
\text { Colonião Tanganika } \\
\text { Grama Jesuita } \\
\text { Catingueiro roxo } \\
\end{array}$ & $\begin{array}{r}4,44 \\
6,12 \\
4,14 \\
13,99 \\
3,38 \\
8,43 \\
5,96 \\
5,79 \\
\end{array}$ & $\begin{array}{l}1,42 \\
0,71 \\
0,95 \\
2,17 \\
1,12 \\
1,40 \\
1,41 \\
0,97\end{array}$ & $\begin{array}{r}13,09 \\
16,01 \\
18,03 \\
11,69 \\
16,28 \\
18,75 \\
9,24 \\
22,41 \\
\end{array}$ & $\begin{array}{l}32,01 \\
31,56 \\
31,49 \\
25,85 \\
38,91 \\
33,91 \\
38,83 \\
35,38 \\
\end{array}$ & $\begin{array}{l}52,73 \\
55,29 \\
55,80 \\
56,41 \\
61,09 \\
64,24 \\
57,20 \\
65,76 \\
\end{array}$ & $\begin{array}{l}1: 10,88 \\
1: 8,00 \\
1: 12,50 \\
1: 3,03 \\
1: 17,10 \\
1: 6,62 \\
1: 8,60 \\
1: 10,30 \\
\end{array}$ & $\begin{array}{l}\text { I. F. } \\
\text { A. } \\
\text { A. F. } \\
\text { N. } \\
\text { P. F. } \\
\text { A. F. } \\
\text { I. F. } \\
\text { N. }\end{array}$ \\
\hline $\begin{array}{l}\text { Jaraguá * } \\
\text { Rhodes * }\end{array}$ & $\begin{array}{l}3,13 \\
3,27\end{array}$ & $\begin{array}{l}1,03 \\
0,67\end{array}$ & $\begin{array}{l}16,15 \\
11,76 \\
\end{array}$ & $\begin{array}{r}20,89 \\
28,64 \\
\end{array}$ & $\begin{array}{l}42,48 \\
45,18\end{array}$ & $\begin{array}{l}1: 12,60 \\
1: 12,81 \\
\end{array}$ & $\begin{array}{l}\text { N. } \\
\text { P. F. }\end{array}$ \\
\hline
\end{tabular}

Resultados obtidos no Laboratório de Bromatologia da 5a. Cadeira

dução Animal de São Queiroz".

Quadro XXIII : Quadro comparativo de resultados obtidos em ensaios com silagens

Silagens $\frac{\text { Nutrientes digestiveis na matéria sêca }(\%)}{n}$ N. D. T. $\quad$ R. N. $\quad$ Ref.

Milho

\begin{tabular}{llllllll} 
Milho & & & & & & & \\
Sta. Rosa & 0,78 & 0,30 & 4,22 & 6,64 & 12,31 & $1: 14,78$ & 1 \\
C. Jaraguá & 1,00 & - & - & $-16,80$ & $1: 15,80$ & 2 \\
C. Jaraguá & $\mathbf{0 , 6 2}$ & $\mathbf{0 , 3 6}$ & $\mathbf{7 , 6 6}$ & $\mathbf{6 , 2 0}$ & 15,29 & $1: 23,60$ & 3 \\
\hline
\end{tabular}

1) Ensaio no laboratório da 5a. Cadeira da E. S. A. "Luiz de Quei-

roz" (12).

2) Dados citados por HODGSON E REED (13), utilizando coeficien-

tes de digestibilidade de forragem semelhante.

Ensaio feito no laboratório da 5a. Cadeira para êste trabalho. 
Quadro XXIV : Composição química do capim jaraguá

\begin{tabular}{|c|c|c|c|c|c|c|c|c|c|c|}
\hline \multirow{2}{*}{$\begin{array}{l}\text { Estágio do } \\
\text { desenvolvimento }\end{array}$} & \multirow{2}{*}{ Forma } & \multicolumn{9}{|c|}{ Composição química (Nutrientes brutos) \% } \\
\hline & & Umid. & M. s. & Prot. & M. G. & Fibra & E. N. N. & Cinza & $\mathrm{CaO}$ & P205 \\
\hline $\begin{array}{l}\text { Novo } \\
\text { Plena floração } \\
\text { Depois da floração } \\
\text { Antes da floração } \\
\text { Antes da floração }\end{array}$ & $\begin{array}{l}\text { Verde } \\
\text { Verde } \\
\text { Verde } \\
\text { Feno } \\
\text { Silagem }\end{array}$ & $\begin{array}{l}70,26 \\
65,74 \\
64,49 \\
13,66 \\
67,80 \\
\end{array}$ & $\begin{array}{l}29,74 \\
34,26 \\
35,51 \\
86,34 \\
32,20 \\
\end{array}$ & $\begin{array}{l}2,73 \\
1,19 \\
0,99 \\
5,63 \\
1,40 \\
\end{array}$ & $\begin{array}{l}0,77 \\
0,65 \\
0,55 \\
2,01 \\
0,82\end{array}$ & $\begin{array}{r}8,58 \\
10,76 \\
11,95 \\
30,21 \\
13,89 \\
\end{array}$ & $\begin{array}{l}13,22 \\
17,00 \\
17,94 \\
33,03 \\
13,16 \\
\end{array}$ & $\begin{array}{r}4,44 \\
4,66 \\
4,08 \\
15,46 \\
2,93 \\
\end{array}$ & $\begin{array}{l}0,16 \\
0,09 \\
0,09 \\
0,53 \\
0,12 \\
\end{array}$ & $\begin{array}{l}0,12 \\
0,05 \\
0,05 \\
0,40 \\
0,06 \\
\end{array}$ \\
\hline
\end{tabular}

Quadro XXV: Digestibilidade e valor nutritivo do capim jaraguá

\begin{tabular}{|c|c|c|c|c|c|c|c|c|c|c|}
\hline \multirow{3}{*}{ Nutrientes } & \multicolumn{6}{|c|}{ Capim Verde } & \multirow{2}{*}{\multicolumn{2}{|c|}{ Fono }} & \multirow{2}{*}{\multicolumn{2}{|c|}{ Silagem }} \\
\hline & \multicolumn{2}{|c|}{ Novo } & \multicolumn{2}{|c|}{ Plena floração } & \multicolumn{2}{|c|}{ Depois da floração } & & & & \\
\hline & $\begin{array}{c}\text { Coef. de } \\
\text { digest. }(\%)\end{array}$ & \begin{tabular}{|c|} 
Nut. digest \\
$(\%)$
\end{tabular} & $\begin{array}{c}\text { Coof. do } \\
\text { digost. }(\%)\end{array}$ & $\begin{array}{c}\text { Nut. digest. } \\
(\%)\end{array}$ & \begin{tabular}{|c|} 
Coef. de \\
digest. $(\%)$
\end{tabular} & \begin{tabular}{|c} 
Nut. digest \\
$(\%)$
\end{tabular} & $\begin{array}{c}\begin{array}{c}\text { Coef. de } \\
\text { digest. }(\%)\end{array} \\
\end{array}$ & $\begin{array}{c}\text { Nut. digest. } \\
(\%)\end{array}$ & $\begin{array}{r}\text { Coef. do } \\
\text { digest. }(\%)\end{array}$ & \begin{tabular}{|c} 
Nut. digent. \\
$(\%)$
\end{tabular} \\
\hline $\begin{array}{l}\text { Matéria sêca } \\
\text { Proteina } \\
\text { Matéria graxa } \\
\text { Fibra } \\
\text { E. N. Nitrogenados } \\
\end{array}$ & $\begin{array}{l}56,58 \\
60,43 \\
56,15 \\
61,92 \\
62,99 \\
\end{array}$ & $\begin{array}{r}16,82 \\
1,64 \\
0,43 \\
5,31 \\
8,32 \\
\end{array}$ & $\begin{array}{l}46,45 \\
25,23 \\
43,75 \\
54,52 \\
52,74 \\
\end{array}$ & $\begin{array}{r}15,91 \\
0,30 \\
0,28 \\
5,86 \\
8,96 \\
\end{array}$ & $\begin{array}{l}42,74 \\
16,46 \\
42,33 \\
47,35 \\
50,23 \\
\end{array}$ & $\begin{array}{r}15.17 \\
0,16 \\
0,23 \\
5,65 \\
9,00 \\
\end{array}$ & $\begin{array}{l}50,57 \\
55,69 \\
51,69 \\
53,46 \\
63,26 \\
\end{array}$ & $\begin{array}{r}43,66 \\
3,13 \\
1,03 \\
16,15 \\
20,89 \\
\end{array}$ & $\begin{array}{l}47,75 \\
44,42 \\
44,60 \\
55,18 \\
47,15\end{array}$ & $\begin{array}{r}15,37 \\
0,62 \\
0,36 \\
7,66 \\
6,20 \\
\end{array}$ \\
\hline $\begin{array}{l}\text { Nutrientes digestiveis } \\
\text { totais } \\
\text { Relação nutritiva } \\
\text { Relação } \mathrm{CaO}: \mathrm{P} 2 \mathrm{O} 5\end{array}$ & & $\begin{array}{c}16,23 \\
1: 8,8 \\
1: 0,75\end{array}$ & & $\begin{array}{c}15,75 \\
1: 51 \\
1: 0,55\end{array}$ & & $\begin{array}{l}15,32 \\
1: 94 \\
1: 0.55\end{array}$ & & $\begin{array}{c}42,48 \\
1: 12,57 \\
1: 0,75\end{array}$ & & $\begin{array}{c}15,29 \\
1: 23,6 \\
1: 0,50\end{array}$ \\
\hline
\end{tabular}

